\title{
PRÁTICAS DE DIAGNÓSTICO PRECOCE DE CÂNCER DE PRÓSTATA ENTRE PROFESSORES DA FACULDADE DE MEDICINA - UFMG
}

\author{
Paulo Sérgio Carneiro Miranda*, Maria da Conceição Juste Werneck Côrtes \\ Maria Efigênia Martins, Patrícia Carolina Chaves, Renata Costa Santarosa \\ Trabalho realizado na Faculdade de Medicina da Universidade Federal de \\ Minas Gerais, Belo Horizonte, MG.
}

\begin{abstract}
RESUMO - OBjetivo. Analisar a prática preventiva frente ao câncer de próstata entre professores-médicos da Faculdade de Medicina da UFMG.

Métodos. Foi realizada uma pesquisa epidemiológica descritiva e quantitativa a partir de um questionário que, preservando 0 anonimato, recolheu informações sobre a prática preventiva frente a este câncer. Distribuído a todos os 289 professores-médicos da FM-UFMG, obtevese resposta de 135 professores-médicos ( $46.7 \%$ do total).

Resultados. Os resultados mais relevantes foram que $20,7 \%$ dos entrevistados com " $5 \mathrm{I}$ e + anos" de idade nunca fizeram nenhum dos dois exames preventivos alvos da pesquisa (toque retal e dosagem de PSA), e que $36,2 \%$ nunca se submeteram à realização do toque retal.
\end{abstract}

Conclusão. Considerando que, até pela função que exercem, a população pesquisada é conhecedora do tema e tem acesso fácil e garantido aos serviços de diagnóstico clínico e complementar, tal resultado merece reflexão não só em relação à categoria como também quanto à população que não tem estes conhecimentos e facilidades. Tal tema seria merecedor de estudos semelhantes em outros grupos da população e da atenção dos responsáveis pela saúde do país.

Unitermos: Câncer de próstata. Médicos. Professores. Prevenção. Rastreamento.

\section{INTRODUÇÃo}

O câncer de próstata é a sexta ocorrência mais freqüente de casos novos de neoplasia maligna no mundo e a terceira causa de morte mais freqüente entre os homens. Representa 9,7\% das neoplasias malignas entre homens (sendo 15,3\%, em países desenvolvidos e 4,3\%, em países em desenvolvimento' É encontrado principalmente acima dos 50 anos e, à medida que a expectativa de vida aumenta, passa a tornar-se mais importante no quadro da mortalidade entre homens ${ }^{2}$.

Especula-se que $\mathrm{o}$ aumento da incidência seja parcialmente devido a uma melhor identificação de casos subclínicos, facilitada pela expansão do uso do teste de PSA (antígeno prostático específico). Por outro lado, o aumento concomitante da mortalidade sugere que o aumento da incidência não pode ser inteiramente explicado por um viés induzido pelo aumento da proporção de casos novos diagnosticados em fase mais precoce.

\footnotetext{
* Correspondência:

Rua Divinópolis, 263 - Apto. 301 - Santa Teresa CEP: 31010-370 - Belo Horizonte - Minas Gerais

Tel.: (31) 3248-9813 - Fax: (31) 3248-9802

E-mail: pmiranda@medicina.ufmg.br
}

Entre 1979 e 2000, a taxa de mortalidade por câncer da próstata, no Brasil, aumentou de 3,73/100.000 para 8,98/100.000. Estimava-se que o número de óbitos ${ }^{9}$ e de casos novos esperados para o ano 2003 seja, respectivamente, de 8230 e 35240 . Estes números esperados correspondem a taxas brutas de mortalidade e incidência de 9,47/100.000 e 40,49//00.000, respectivamente ${ }^{3}$.

Em Minas Gerais, o tumor de próstata ocupa o segundo lugar entre os tumores mais comuns e o terceiro lugar entre os mais letais no sexo masculino ${ }^{4}$. Em um estudo realizado sobre o perfil de mortalidade entre os médicos de Belo Horizonte, nos anos de 1979 a 1995, o câncer de próstata apareceu como terceira causa de morte, tendo assim peso considerável no perfil de mortalidade dos médicos $^{5}$.

O câncer de próstata é uma patologia que pode ser detectada precocemente através de métodos diagnósticos de triagem. De acordo com a Sociedade Americana de Cancerologia, para a detecção precoce do câncer em indivíduos sem sintomas preconiza-se o toque retal e o PSA sérico anuais a partir de 50 anos de idade $^{6}$. Estes exames, além do baixo custo, possuem boa sensibilidade e especificidade. Inclusive, estudos recentes sugerem que a triagem de homens em idade acima de 50 anos através do toque retal e do PSA diminuiu a incidência de doença tardia com influência nas taxas de mortalidade ${ }^{7,8}$, na medida em que 0 câncer de próstata pode ser curável, desde que diagnosticado precocemente.

O conhecimento da patologia e o acesso aos serviços preventivos e de diagnósticos são considerados pontos chaves na prática preventiva. Conhecendo-se a evolução do câncer de próstata, os métodos de diagnóstico precoce e dispondo-se de condições de acesso aos serviços médicos-laboratoriais, potencialmente o câncer de próstata pode ser detectado numa fase inicial e com isto o caso apresentar, na maioria das vezes, melhor prognóstico

Sendo o câncer de próstata o que mais incide em homens, e tendo-se constituído na terceira principal causa de morte entre os médicos, achamos necessária uma investigação para avaliar se os professores-médicos, como profissionais da saúde e da educação, encarregados de informar e sensibilizar a população a respeito do diagnóstico precoce e tratamento do câncer de próstata, estão ou não usando seus conhecimentos para si mesmos assim como utilizando da possibilidade de acesso mais fácil a serviços de saúde para a sua prevenção. $O$ resultado encontrado 
expressará o nível de postura preventiva frente ao câncer de próstata entre uma categoria privilegiada pelo acesso ao conhecimento e aos serviços de saúde.

Portanto, o objetivo desta investigação é mensurar o uso e avaliar a aceitação dos métodos diagnósticos de triagem (toque retal e dosagem de PSA) de câncer de próstata entre os professores médicos da Faculdade de Medicina - UFMG, cadastrados no ano de 2000. A partir dos resultados deste estudo, pretende-se sensibilizar os médicos e professores médicos a respeito do seu comportamento em relação à doença e do lugar importante que esta ocupa no perfil de mortalidade destes profissionais, promovendo discussão sobre o assunto. Outra finalidade é refletir como estará a prevenção de câncer de próstata entre homens que não dispõem da mesma condição de acesso a serviços de saúde e informações sobre as doenças.

\section{Métodos}

O estudo foi realizado entre todos os professores-médicos da Faculdade de Medicina (FM-UFMG), no ano de 2000, através de questionário no qual se pedia a participação de todos e se garantia o anonimato do participante. Para isto foram elaboradas questões que identificavam o departamento do professor e sua faixa etária; seu comportamento preventivo em relação ao câncer de próstata (idade do primeiro exame de toque retal e de realização do PSA e a freqüência dos mesmos) e sobre a existência de câncer de próstata entre familiares de primeiro grau. Em seguida foram os questionários distribuídos a todos os 289 professores-médicos (homens), em atividade, da Faculdade de Medicina - UFMG, no ano de 2000. Este total de professores-médicos foi fornecido pela Seção de Ensino da Faculdade, considerando sua distribuição pelos departamentos da Faculdade, em junho de 2000. Os questionários foram colocados nos escaninhos dos professores e foi solicitado aos mesmos que após respondê-los fossem deixados no escaninho do chefe do departamento, que os repassou aos investigadores. Os professores não médicos da Faculdade foram excluídos da pesquisa. A partir de então, montou-se um banco de dados e foram feitas as análises pelo programa Epi Info 6.0, através de freqüências e tabelas $2 \times 2$, considerando um valor de $p<0,05$ como significativo.

Tabela I - Professores do Curso Médico, do sexo masculino, por departamento: número total, amostrados e cobertura - Faculdade de Medicina da UFMG - Belo Horizonte - Junho - Dezembro/2000

\begin{tabular}{lcccc}
\hline Departamento & $\begin{array}{c}\text { Prof. do sexo } \\
\text { masculino }\end{array}$ & & $\begin{array}{c}\text { Professores } \\
\text { amostrados }\end{array}$ & $\begin{array}{c}\% \\
\text { Amostrados/Total }\end{array}$ \\
\cline { 2 - 2 } Clínica Médica & 67 & & 22 & \\
Aparelho Locomotor & 09 & & 03 & 33.0 \\
Anatomia Patológica & 09 & 05 & 33.3 \\
Cirurgia & 64 & 26 & 55.5 \\
Ginecologia-Obstetrícia & 19 & 10 & 40.6 \\
Medicina Preventiva & 26 & 18 & 52.6 \\
Oftalmo-Otorrino & 19 & 19 & 69.2 \\
Proped. Complementar & 16 & 06 & 100.0 \\
Pediatria & 40 & 16 & 37.5 \\
Psiquiatria-Neurologia & 20 & 10 & 40.0 \\
Total & 289 & 135 & 50.0 \\
\hline
\end{tabular}

Fonte: Seção de Ensino - Junho de 2000 e Pesquisa de Campo

\section{Tabela 2 - Professores do Curso Médico, do sexo masculino por faixa etária: número total e porcentagem, número amostrado e porcentagem do total - Faculdade de Medicina da UFMG - Belo Horizonte - Junho - Dezembro/2000}

\begin{tabular}{|c|c|c|c|c|}
\hline Faixa Etária & $\begin{array}{l}\text { Professores } \\
\text { Total } \\
\text { Número }\end{array}$ & $\begin{array}{l}\text { Professores } \\
\text { Total } \\
\text { Porcentagem }\end{array}$ & $\begin{array}{c}\text { Professores } \\
\text { Amostrados } \\
\text { Número }\end{array}$ & $\begin{array}{l}\text { Amostra/Total } \\
\text { Porcentagem }\end{array}$ \\
\hline $\begin{array}{c}</=30 \\
3 \mid-35 \\
36-40 \\
4 \mid-45 \\
46-50 \\
>\mid=51 \\
\text { Total }\end{array}$ & $\begin{array}{l}02 \\
09 \\
24 \\
27 \\
94 \\
133 \\
289\end{array}$ & $\begin{array}{c}0.7 \\
3.1 \\
8.3 \\
9.3 \\
32.5 \\
46.1 \\
100.0\end{array}$ & $\begin{array}{l}0 \\
06 \\
16 \\
19 \\
36 \\
58 \\
135\end{array}$ & $\begin{array}{l}0.0 \\
66.6 \\
66.6 \\
70.0 \\
38.3 \\
43.6 \\
46.7\end{array}$ \\
\hline
\end{tabular}

Fonte: Seção de Ensino - Junho/2000 e Pesquisa de Campo

\section{REsultados}

Responderam ao questionário um total de 135 (46,7\% do universo) dos 289 professores médicos em atividade na Faculdade em junho de 2000

Nas Tabelas I e 2 se apresenta como se distribuía o universo e como se distribuiu a amostra estudada, destacando-se a representatividade da mesma.

Quando solicitadas informações sobre práticas preventivas, independentemente da prática, freqüência e idade do infor mante, verificou-se que $64,4 \%$ dos professores afirmaram a prática preventiva e $35,6 \%$ a negaram.

Procurou-se refinar a análise destacando-se a prática de exames preventivos entre os professores de " 5 I ou mais anos de idade", pois a partir desta idade, os especialistas concordam - apesar das limitações que podem haver na prática diagnóstica e preventiva de câncer de próstata, através do toque retal e da dosagem do PSA - na sua orientação e sua utilidade. As relações que apresentaram significação estatística estão descritas nas tabelas que se seguem e são comentadas na Tabela 3.

Observou-se que 20,7\% dos professores médicos da Faculdade de Medicina, com idade de "5I anos e mais" nunca fizeram um toque retal e uma dosagem de PSA como prática de detecção precoce do câncer de próstata e $36,2 \%$ nunca se submeteram a um toque retal, com o mesmo objetivo.

Dos 135 médicos professores que responderam aos questionários, 12 (8,6\%) apresentavam parente de primeiro grau que tiveram ou têm câncer de próstata.

Destes 12, um total de cinco professores médicos estavam com "5I anos e mais". 


\begin{tabular}{lcc}
\hline $\begin{array}{c}\text { Tabela 3 - Prática diagnóstica e preventiva de câncer de próstata entre } \\
\text { professores do curso médico, do sexo masculino e com 5I e mais anos de idade }\end{array}$ \\
por tipo de exame - Faculdade de Medicina da UFMG - Belo Horizonte - Junho - Dezembro/2000 \\
\hline Tipo de exame realizado & $\frac{\text { Número Absoluto }}{\text { Número Percentual }}$ \\
\cline { 2 - 3 } Toque retal + PSA & 36 & 62.1 \\
Só toque retal & 01 & 1.7 \\
Só PSA & 09 & 15.5 \\
Não (Toque retal + PSA) & 12 & 20.7 \\
Total & 58 & 100.0 \\
\hline
\end{tabular}

Fonte: Pesquisa de Campo - FMUFMG - Junho-Dezembro de 2000

\section{Tabela 4 - Professores do curso médico, do sexo masculino e com $5 \mathrm{I}$ e mais anos de idade que nunca se submeteram ao toque retal como prática diagnóstica e preventiva de câncer de próstata segundo justificativa apresentada - Faculdade de Medicina da UFMG - Belo Horizonte - Junho - Dezembro/2000}

\begin{tabular}{l} 
Justificativa apresentada para não \\
submeter-se ao Toque Retal \\
\hline Medo \\
Preconceito \\
Falta de tempo \\
Ser muito saudável \\
Descuido \\
Confiança no Exame de PSA \\
Não ter história familiar \\
Outros \\
Total
\end{tabular}

Número Absoluto

Fonte: Pesquisa de Campo

Obs: * As alternativas não eram excludentes

Destes cinco, três se submetiam aos exames preventivos do Toque Retal + PSA, mas na freqüência de dois em dois anos. Um dos cinco havia feito somente PSA, e um outro professor médico, apesar da história familiar e da idade nunca havia feito Toque Retal + PSA.

Havia ainda dois outros professores com história familiar de câncer de próstata na idade de 46 a 50 anos. Destes dois professores, um deles se submetia a exames preventivos de câncer de próstata através do Toque Retal + PSA, porém, na freqüência de dois em dois anos. O outro só realiza a dosagem de PSA, e com freqüência de dois em dois anos.

Como se pode observar, mesmo na idade de " 5 I anos e mais" encontra-se um total de $36,2 \%$ de professores médicos (21/58) que nunca se submeteram ao toque retal, tendo ou não historia de câncer de próstata na família.

Procurou-se analisar a periodicidade do toque retal entre os professores-médicos de "5I e mais anos" e encontrou-se que, dos 58 profissionais nesta faixa etária, somente três ( $5, \mid$ I\%) se submetiam ao toque retal anual, como prática preventiva para câncer de próstata.

$\mathrm{Na}$ análise da periodicidade do teste do PSA nesta mesma faixa etária, encontrou-se que dos 58 professores-médicos somente 10 $(17,2 \%)$ se submetiam ao PSA anualmente.

Entre aqueles 36 professores-médicos de " 5 I anos e mais" que realizam os dois exames (Toque Retal + PSA), apenas 8,3\% (3/36) fazem o toque retal anualmente e $16,6 \%$ (6) 36) fazem o PSA anualmente.

Em síntese, mesmo entre os 36 (62\%) professores-médicos de 51 e + anos que cumprem o protolocolo de realização de ambos os exames, somente uma pequena parcela $3 / 58$ (5.2\%) realiza os exames na prescrição estabelecida.

$\mathrm{Na}$ Tabela 4, se apresentam as justificativas apresentadas pelos professores-médicos de "5 I e + anos de idade" para a não realização do Toque Retal como prática preventiva para câncer de próstata.

\section{Discussão}

Sendo a discussão o exame atento e particular de uma matéria, dois pontos se destacariam dos resultados e mereceriam este aprofundamento.

O primeiro é relativo ao percentual de professores-médicos da Faculdade de Medicina que, estando na faixa etária de 51 e mais anos, nunca realizaram práticas preventivas para câncer de próstata, apesar de serem, até pela função que exercem, conhecedores do tema, terem acesso fácil e garantido aos serviços de diagnóstico clínico e complementar. Conhecimento das informações e acesso aos serviços de saúde são freqüentemente citados como os impeditivos básicos à realização das práticas preventivas entre a população. Estas limitações os professores-médicos da Faculdade não sofrem.

O segundo ponto que mereceria o exame atento e particular da matéria é o constituído pelas justificativas apresentadas pelos professores-médicos para a não realização do exame de toque retal como preventivo de câncer de próstata.

Quanto ao primeiro ponto, tivemos grandes dificuldades para encontrar artigos ou outras publicações que tratassem da especificidade estudada nesta pesquisa. Em consulta aos bancos de dados MEDLINE e LILACS, não se encontrou referencias de trabalhos que tratassem do objeto específico deste trabalho. Torna-se, portanto, temerário afirmar que $20 \%$ da amostra de professores-médicos com "5I anos e mais de idade" que não realizam exames preventivos para a câncer de próstata é o número esperado para esta população ou que este percentual é muito baixo ou muito alto. Estudos comparativos a serem realizados com este tipo de população usando metodologia semelhante poderiam contribuir para confirmar ou reforçar alguma das afirmações. Acredita-se ser este percentual preocupante e digno de aprofundamento das investigações pelos fatores comentados nesta discussão.

O que reforça a necessidade deste aprofundamento é que investigações conduzidas em médicas norueguesas com relação à prevenção do câncer de mama e colo de útero mostram que a prática preventiva do autoexame não é realizada por 19,2\% das médicas norueguesas; e que ao exame de Papanicolaou 
nunca se submeteram $16,02 \%$ delas $^{9}$. Entre as médicas alemãs, o auto-exame das mamas é realizado por $86 \%$ delas sendo que $14 \%$ afirmam que nunca o realizaram ${ }^{10}$. Ou seja, considerando o perfil dos médicos e médicas e o grau de sensiblidade/especificidade de um auto-exame das mamas e do toque retal poderíamos levantar a hipótese (por analogia) que a categoria médica (ainda que no presente estudo sejam médicos-professores) tem uma postura semelhante quanto ao seu auto-cuidado. $\mathrm{O}$ que, ainda que seja um novo ponto para reflexão, não acrescenta muito à especificidade da investigação atual que se apresenta.

Em um estudo exploratório prévio a esta pesquisa, verificou-se a referência à prática de exame preventivo de câncer de próstata entre professores e servidores técnicos-administrativos da Universidade Federal de Minas Gerais, que realizaram exame periódicos previstos pela instituição. Em 31 professores com 51 e mais anos de idade, 26 ( 83\%) afirmaram que haviam feito exame preventivo. Este dado deve ser relativizado, pois é a única questão nos formulários usados pelo Serviço de Assistência da Saúde do Trabalhador (SASTUFMG) relativa ao tema. Não há especificação sobre o que se define como prevenção de câncer de próstata (Toque Retal e/ou Dosagem de PSA), sua freqüência, idade ao primeiro exame, câncer na família. Entre os funcionários técnicos-administrativos da UFMG analisados nesta amostra e neste grupo etário, a freqüência de realização de exame preventivo cai para $20 \%$, ou seja, I5 afirmaram que realizaram exame preventivo para câncer de próstata e 60 não o realizaram.

O segundo ponto que mereceria o estudo atento são as justificativas sobre a não realização do toque retal. As apresentadas na presente investigação falam, em primeiro lugar, do processo de trabalho médico que Ihes dificultaria o uso dos serviços de diagnóstico. O segundo conjunto de justificativas se centra na própria pessoa do médico que se expressa como "descuidado" ou auto-confiante em sua saúde. A questão do preconceito em relação exame não se apresenta como justificativa dominante. Dentro da realidade brasileira, a pouca referência a esta justificativa constituiria-se mesmo num "preconceito" e poder-se-ia ser motivo de investigação. Alexandrina Meleiro nos fala do comportamento do médico como paciente, sua dificuldade de se aceitar como um ser comum sujeito às patologias e seus mecanismos de defesa para enfrentar a ameaça do adoecer ${ }^{\prime \prime}$. Um estudo qualitativo, através de entrevistas profundas com médicos, poderia contribuir para a compreensão destas posturas contraditórias entre o conhecimento do médico e sua prática quando ele é o paciente.

\section{Conflito de interesse: não há.}

\section{SUMMARY}

Practice of precocious diagnosis for PROSTATE CANCER AMONG PROFESSORS OF the school of medicine, Minas Gerais Federal University- Brazil

BACKGROUD. The authors studied the practice of precocious diagnosis for prostate cancer among 289 professor-physicians of the School of Medicine, Minas Gerais Federal University, Brazil.

Methods. Replies on these preventive practices were received from 135 professorphysicians through a confidential questionnaire administered.

RESULTS. Results show that $20.7 \%$ of the professor-physicians "fifty-one years old or more" never had a prostate preventive exam and $36.2 \%$ never had a rectal touch.

Conclusions. This data suggests the need for additional investigation and the attention of the health services. [Rev Assoc Bras 2004; 50(3): 272-5]

KEY WORDS: Prostate cancer. Physicians. Professors. Prevention. Screening.

\section{REFERÊNCIAS}

I. Parkim DM, Bray FI, Devesa SS. Cancer Burden in the year 2000. The Global picture. Eur J Cancer 200 I; 37:54-66.

2. INCA. Instituto Nacional do Câncer. Estimativa da incidência e mortalidade por câncer. Rio de Janeiro: Ministério da Saúde; 2002. p. I-4.

3. INCA. Instituto Nacional do Câncer. Estimativa da incidência e mortalidade por câncer [online]. Ministério da Saúde; 2003. Disponível em: http://www.inca.gov.br/estimativas/ 2003/conteúdo.

4. Siqueira M. Câncer de pulmão já é o que mais mata. Estado de Minas, Belo Horizonte. 2000 abr 25: 28

5. Arcanjo HR, Silva AC, Miranda PSC, Pinheiro TMM. Estudo da morbi-mortalidade entre médicos da Região Metropolitana de Belo Horizonte, Minas Gerais. Considerações especiais sobre o suicido neste grupo de trabalhadores. Rev Med Minas Gerais 2000; 10:72-7.

6. Tierney LM, McPhee SJ, Papadakis A. Current diagnose and treatment. Norwalk: Prentice Hall; 1 996. p.224-7.

7. Smart CR. The results of prostate carcinoma screening in the U.S. as reflected in the surveillance, epidemiology and end results program. Cancer 1997; 80: 1835-44.

8. Merrill RM, Stephenson RA. Trends in mortality rates in patents with prostate cancer during the era of prostate specif antigen screening. J Urol 2000; 163:503-10.

9. Rosvold EO, Hjartaker A, Bjertness E, Lund E. Breast self-examination and cervical cancer testing among Norwegian female physicians. A nation-wide comparative study. London. Soc Sci Med 200 I ; $52: 249$ 58.

10. Rummler S, Schimpf H. Zu einigen Aspekten des Gesundheitsverhaltens von Arztinnen. Zeitschrift Arztl Fortbildung 1990; 84:559-61.

1 ।. Meleiro AMAS. O médico como paciente. São Paulo: Lemos Editorial; 1999. p.82-4.

Artigo recebido: 10/09/2003

Aceito para publicação: 0 I/03/2004 